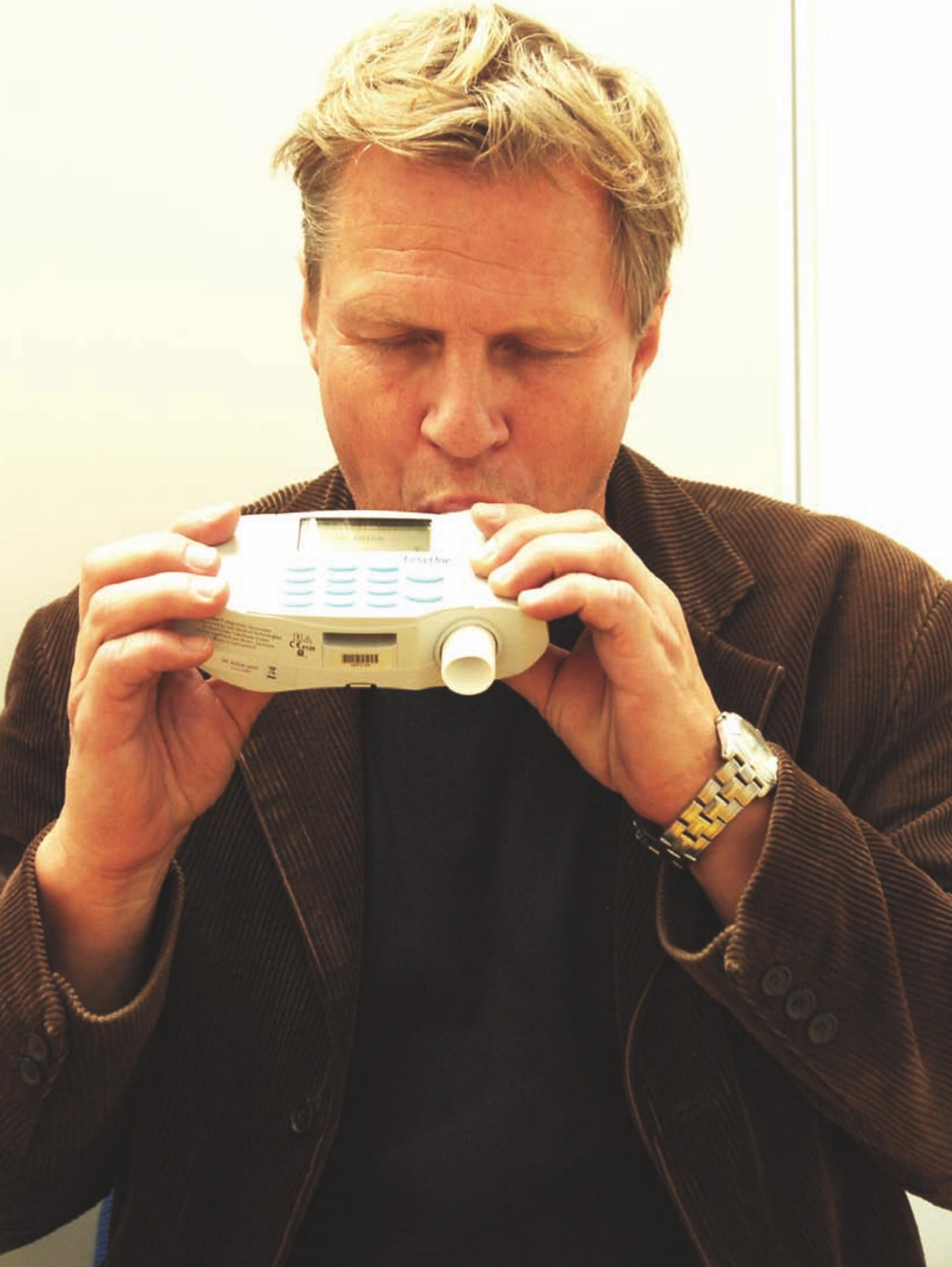




\begin{tabular}{|c|c|c|c|}
\hline$\Omega$ & (1] & $\equiv$ & (2) \\
\hline V.C. Moore & $\begin{array}{l}\text { Occupational Lung Disease } \\
\text { Unit, Dept of Respiratory } \\
\text { Medicine, Birmingham } \\
\text { Heartlands Hospital, } \\
\text { Birmingham, UK }\end{array}$ & $\begin{array}{l}\text { Occupational Lung Disease } \\
\text { Unit, Dept of Respiratory } \\
\text { Medicine, Birmingham } \\
\text { Heartlands Hospital, } \\
\text { Bordesley Green East, } \\
\text { Birmingham, B9 } 5 \text { SS, UK }\end{array}$ & vicky.c.moore@heartofengland.nhs.uk \\
\hline
\end{tabular}

\section{Spirometry: step by step}

\section{Educational aims}

- To give an overview of what spirometry is, how to perform it and how to interpret it.

- To discuss the quality criteria to produce acceptable and reproducible results.

- To discuss the use of spirometry in the workplace.

\section{Summary}

Spirometry is easy to perform once trained and can be performed anywhere. It is useful for detecting early change and disease progression. Quality is important to ensure useful and reproducible results, otherwise results may be incorrectly interpreted. Training from a reputable centre should be undertaken to ensure the measures are understood as well as how to get the best results out of the patient.

\section{Introduction}

Spirometry is the term given to the basic lung function tests that measure the air that is expired and inspired. There are three basic related measurements: volume, time and flow. Spirometry is objective, noninvasive, sensitive to early change and reproducible. With the availability of portable meters it can be performed almost anywhere and, with the right training, it can be performed by anybody. It is performed to detect the presence or absence of lung disease, quantify lung impairment, monitor the effects of occupational/ environmental exposures and determine the effects of medications.

\section{The measures}

Spirometric measures include the following.

- Forced expiratory volume in $1 \mathrm{~S}\left(\mathrm{FEV}_{1}\right)$

- Forced vital capacity (FVC), the maximum amount of air that can be exhaled when blowing out as fast as possible

- Vital capacity (VC), the maximum amount of air that can be exhaled when blowing out at a steady rate

- $\mathrm{FEV}_{1} / \mathrm{FVC}$ ratio

- Peak expiratory flow (PEF), the maximal flow that can be exhaled when blowing out as fast as possible
Statement of Interest None declared.
This article has been corrected according to the erratum published in the December 2012 print issue of Breathe

HERMES syllabus links: A.1.2, B.1.1, B.1.4, B.6.1, D.1.1 
- Forced expiratory flow, also known as midexpiratory flow; the rates at $25 \%, 50 \%$ and $75 \% \mathrm{FVC}$ are given

- Inspiratory vital capacity (IVC), the maximum amount of air that can be inhaled after a full expiration

\section{Performing spirometry Calibration}

Before performing spirometry, the equipment used must be calibrated, or at least the calibration checked at the beginning of the session. Depending on the type of equipment, this is achieved using either a $3-L$ syringe that is pumped through to check that the meter is reading correctly (within a tolerance of $3 \%$ ) or using a $\mathrm{I}-\mathrm{L}$ syringe that is pumped a litre at a time to a maximum of $7 \mathrm{~L}$, which checks the linearity as well as the centre point of the volume measurement. Many spirometers also allow linear calibration, i.e. the volume is checked at different flows. Some portable meters do not require calibration, for example those that use ultrasound technology. With many meters, the calibration is a checking function and if the calibration is out, the meter needs to be returned to the manufacturer for

Table 1 Checklist of factors that the patient should avoid prior to spirometry

\begin{tabular}{lc} 
Activity & $\begin{array}{c}\text { Length of time to } \\
\text { stop before } \\
\text { spirometry }\end{array}$ \\
\hline Drinking alcohol & $4 \mathrm{~h}$ \\
\hline Eating a large meal & $2 \mathrm{~h}$ \\
\hline Vigorous exercise & $30 \mathrm{~min}$ \\
\hline Smoking & $>_{1 \mathrm{~h}}$ \\
\hline Medication use & $\begin{array}{c}\text { Document } \\
\text { treatment and when } \\
\text { last taken }\end{array}$ \\
\hline For reversibility testing & \\
\hline
\end{tabular}

Taking short-acting bronchodilators

$6 \mathrm{~h}$

Taking long-acting bronchodilators (including

combination inhalers) or twice-daily preparations

Taking tiotropium or once-daily preparations repair. There is an exception to this where some of the more sophisticated equipment, such as you would find in a lung function laboratory, can update its output based on the calibration. Spirometric values should also be checked on a weekly basis using a biological control (a healthy person working in the laboratory).

Flow is very difficult to calibrate and is not calibrated routinely. It requires a sophisticated computer-driven syringe to reproduce forced expiration.

\section{Prior to testing}

Prior to performing spirometry, the patient's identification should be checked, their height without shoes or boots and weight measured (if scales are available, as this is not used in prediction equations but is useful to know, as volume may be restricted in obese patients), and their age, sex and race recorded. If the patient is unable to stand to have their height measured, arm span can be used as an estimate. Table 1 gives a checklist of what should be avoided by the patient before spirometry.

If inhalers have been taken within the times stated above when reversibility testing, a true assessment may not be made as the effect of the administered therapy will be weakened. For general testing, however, normal medication should be documented so that it is known what the patient's lung function is like on and off therapy, and if spirometry is going to be repeated over time, conditions can be kept the same. Many centres perform longitudinal measurements post-bronchodilator to minimise variability in recent bronchodilator use.

\section{Contraindications}

If any of the following have occurred recently, then it may be better to wait until the patient has fully recovered before carrying out spirometry.

- Haemoptysis of unknown origin

- Pneumothorax

- Unstable cardiovascular status, recent myocardial infarction or pulmonary embolism

- Thoracic, abdominal or cerebral aneurysms

- Recent eye surgery

- Acute disorders affecting test performance, such as nausea or vomiting

- Recent thoracic or abdominal surgical procedures 


\section{Patient positioning}

Correct measurement posture is as follows.

- Sit upright: there should be no difference in the amount of air the patient can exhale from a sitting position compared to a standing position as long as they are sitting up straight and there are no restrictions.

- Feet flat on floor with legs uncrossed: no use of abdominal muscles for leg position.

- Loosen tight-fitting clothing: if clothing is too tight, this can give restrictive pictures on spirometry (give lower volumes than are true).

- Dentures normally left in: it is best to have some structure to the mouth area unless dentures are very loose.

- Use a chair with arms: when exhaling maximally, patients can become light-headed and possibly sway or faint.

\section{Infection control}

Hands must be washed between patients. Bacterial-viral filters should be used for all patients and thrown away by the patient at the end of testing. If an infectious patient requires testing, this should be performed at the end of the session and the equipment should be stripped down and sterilised/parts replaced (depending on what is being used) before being used again.

\section{Technique}

There are a number of different techniques for performing spirometry.

- Before performing the forced expiration, tidal (normal) breaths can be taken first, then a deep breath taken in while still using the mouthpiece, followed by a further quick, full inspiration.

- Alternatively, a deep breath can be taken in then the mouth placed tightly around the mouthpiece before a full expiration is performed.

- The patient can be asked to completely empty their lungs then take in a quick full inspiration, followed by a full expiration.

The latter technique can be useful in patients who may achieve a larger inspiration following expiration.
- For FVC and $\mathrm{FEV}_{1}$, the patient takes a deep breath in, as large as possible, and blows out as hard and as fast as possible and keeps going until there is no air left.

- PEF is obtained from the FEV 1 and FVC manoeuvre.

- For VC, the patient takes a deep breath in, as large as possible, and blows steadily for as long as possible until there is no air left. Nose clips are essential for VC as air can leak out due to the low flow.

- The IVC manoeuvre is performed at the end of FVC/VC (depending on what type of equipment is used) by taking a deep, fast breath back in after breathing all the way out.

Encouragement makes a big difference, so don't be afraid to raise your voice to encourage the patient, particularly near the end of the manoeuvre. The patient needs to keep blowing until no more air comes out and the volumetime trace reaches a plateau with $<50 \mathrm{~mL}$ being exhaled in $2 \mathrm{~s}$ [1]. Some patients, particularly those with obstructive disease, may find it difficult to exhale completely on a forced manoeuvre. A relaxed VC may produce better results in this case.

\section{Quality}

A number of criteria for acceptable quality spirometry have been published. Guidelines from the American Thoracic Society (ATS)/ European Respiratory Society (ERS) Task Force [1] suggest that three acceptable manoeuvres should be achieved. An acceptable manoeuvre is defined as follows.

- An explosive start (no hesitation or sigmoid curve) with a back-extrapolation volume $<150 \mathrm{~mL}$ (fig. 1) [1].

- The manoeuvre was performed with a maximal inspiration and expiration.

- No glottis closure or cessation of airflow occurred during the manoeuvre (e.g. by hesitation or blocking the mouthpiece).

- No coughs (particularly during the first second), inspirations during the trace or evidence of leaks.

- The manoeuvre should meet the end-of-test criteria (exhaling for $\geqslant 6 \mathrm{~s}$ with $<50 \mathrm{~mL}$ being exhaled in the last $2 \mathrm{~s}$ ).

The best two measurements must fulfil the reproducibility criteria. 


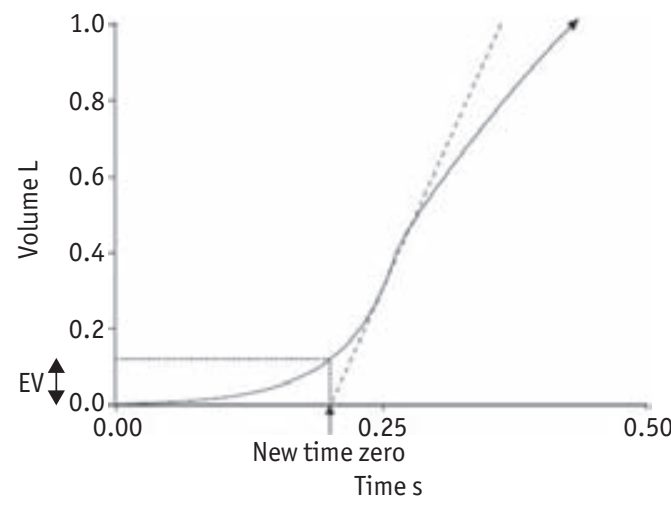

Figure 1

Expanded version of the early part of a subject's volume-time spirogram, illustrating back-extrapolation through the steepest part of the curve, where flow is peak expiratory flow (PEF), to determine the new time zero. Forced vital capacity (FVC): 4.291 L; back extrapolated volume (EV): 0.123 L (2.9\% FVC). -----: back extrapolation line through PEF. Reproduced from [1] with permission from the publisher.

- For $\mathrm{FEV}_{1}$ and FVC, the best two values should be within $5 \%$ or $150 \mathrm{~mL}$ of each other, whichever is greater. If FVC is $<1.0 \mathrm{~L}$, then the values should be within $100 \mathrm{~mL}$.

- The best FEV 1 and FVC can be taken from different manoeuvres.

If this is not achieved, more manoeuvres are required. There is usually an upper limit of eight manoeuvres, as performing forced exhalations is extremely tiring and the patient is unlikely to obtain better values after this point.

There can be problems with reproducibility: forced expiration can cause bronchoconstriction, so there must be $\geqslant 30 \mathrm{~s}$ between manoeuvres. For some patients, particularly asthmatics, several minutes may need to be left. However, full inspiration can cause bronchodilation, so it is important to keep going until there is no further improvement.

\section{BTPS correction}

Spirometry should be corrected for body temperature and ambient pressure saturated with water vapour (BTPS). This is to correct for the difference in the volume of air in the lungs (at $37^{\circ} \mathrm{C}$ ) to the volume measured by the spirometer (at room air temperature). Where barometric pressure cannot be inputted, the spirometer should have a pressure range for the correction factor used.

\section{Reversibility testing}

Reversibility testing is usually performed for the diagnosis of asthma. Spirometry is performed, after which a bronchodilator is given that can either be a short-acting $\beta$-agonist or other agents, such as anticholinergics. For the former, $4 \times 100 \mu \mathrm{g}$ salbutamol are recommended via a spacer device and $15 \mathrm{~min}$ is given before retesting, and for the latter, $4 \times 40 \mu \mathrm{g}$ ipratropium bromide is recommended, leaving $30 \mathrm{~min}$ before retesting [1]. There are a number of publications [2-5] that all have slightly different criteria for what constitutes a significant improvement in lung function to determine that the individual has reversed. The combined ATS/ ERS Task Force [4] recommends that there should be at least a $12 \%$ from baseline and $200-$ $\mathrm{mL}$ improvement in $\mathrm{FEV}_{\mathrm{r}}$. The British Thoracic Society (BTS)/Association of Respiratory Technicians and Physiologists (ARTP) guidelines suggest $>160 \mathrm{~mL}$ for $\mathrm{FEV}_{1}$ and $>330 \mathrm{~mL}$ for FVC [5]. These measures of reversibility show that the change is outside the $95 \%$ confidence limits for repeat spirometry without any bronchodilator [6] and do not relate to diagnosis or usefulness of the drug in everyday use. Reversibility is the defining characteristic of asthma and it separates asthma from other causes of airflow obstruction. An increase of $\geqslant 400 \mathrm{~mL}$ in $\mathrm{FEV}_{1}$ post-bronchodilator is strongly suggestive of asthma in adults, but a lack of reversibility does not exclude asthma [2].

\section{Equipment}

A number of spirometers are available, ranging from desktop portable devices to large, less portable versions. Most measure flow directly using pneumotachographs, turbines or other technology and calculate volume, but the wedge bellows spirometer measures volume directly and calculates flow. The choice of equipment depends on your service needs. Larger equipment tends to be more stable, but there are now some very usable hand-held devices that store flow-volume loops, have built-in quality control and measure all spirometric indices. Some of these have printing capacity as well, whereas others require attachment to a computer for this.

\section{Interpreting spirometry Normal lung function}

Results obtained from lung function tests have no meaning unless they are compared against 
reference values or predicted values. There are a number of reference values available that have been equated in slightly different ways, but for studies comparing different European communities, the equations from the European Community for Coal and Steel (ECCS) are often used [7]. Reference values are derived from reference equations that contain data from population surveys. The population in the survey is ideally very large, and data is gathered about the subjects' height, weight, age, sex, ethnic origin, smoking habits, environment, working conditions and physical fitness. The current ECCS equations are linear in nature but in reality, lung function change is a nonlinear process. Reference values given can, therefore, be unrepresentative of the person being tested in some situations. There will be new equations available in the near future which should overcome these issues.

In adults, age, height, sex and race are the main determinants of the reference values for spirometric measurement.

- Age Lung function generally increases with age up to $\sim 25$ years, then declines with increasing age afterwards. Unfortunately, some lung function equipment will give patients aged $<25$ years larger predicted values than at age 25 years. To avoid majorly overestimating the predicted value for patients $<25$ years of age, the best course of action is input the subject's age as 16 years and then 25 years. If the predicted is larger at 16 years, then use the value for 25 years.

- Sex Pre-pubescent males and females generally have the same lung function, but postpuberty, the growth of the thorax is greater in males, giving marked differences in lung volumes.

- Height The taller the person, the bigger the lungs.

- Weight Certain reference equations use weight to calculate reference values. Weight affects lung function in that increasing weight causes increasing lung function until obesity is reached, after which it has the opposite effect.

- Ethnic origin This factor becomes more difficult to include as a multiethnic society develops. The BTS/ARTP guidelines suggest that for Japanese, Polynesian, Indian, Pakistani and African patients, and those of African descent, reference values multiplied by a factor of 0.90 should be used [5]. This is

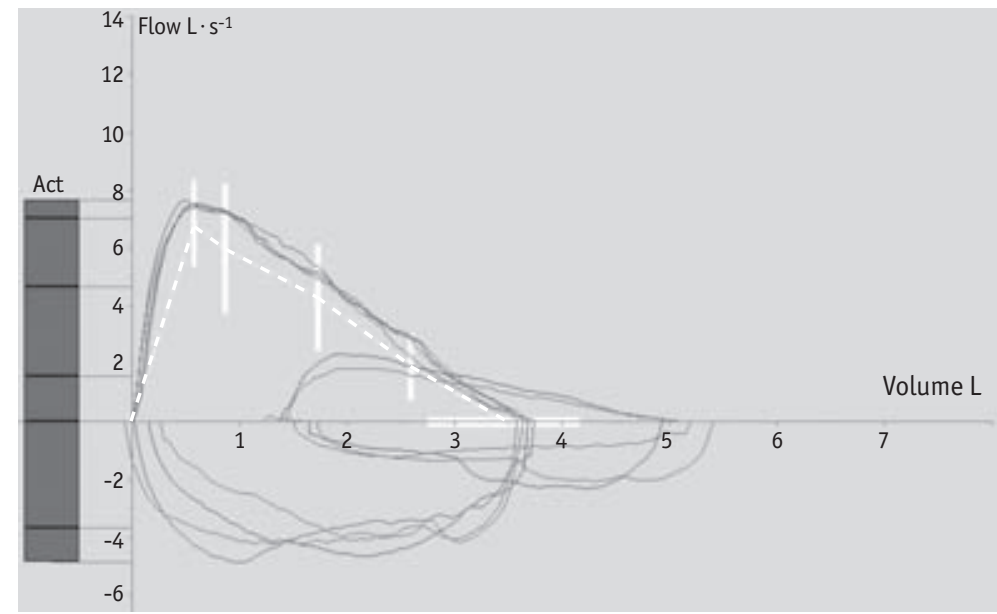

Figure 2

Flow-volume loop of a normal subject. Forced expiratory volume in $1 \mathrm{~s}$ (FEV 1 ): 105\% predicted; forced vital capacity (FVC): 103\% pred; FEV 1 FVC ratio: $89 \%$. The plot shows the predicted flow-volume loop (----) and predicted ranges for the peak and mid-expiratory flow rates and the FVC.

due either to body shape (i.e. African people tend to have longer legs and shorter bodies than Caucasians) or lack of nutrition for those born in poorer countries. As many different ethnicities are now born in richer countries, nutrition is likely to be less of an issue and as a general rule, by the second generation after immigration, adjustment for race is not usually required. Flow measurements are not affected.

- Smoking This can cause a more rapid decline in lung function compared with nonsmokers over time. It should not be

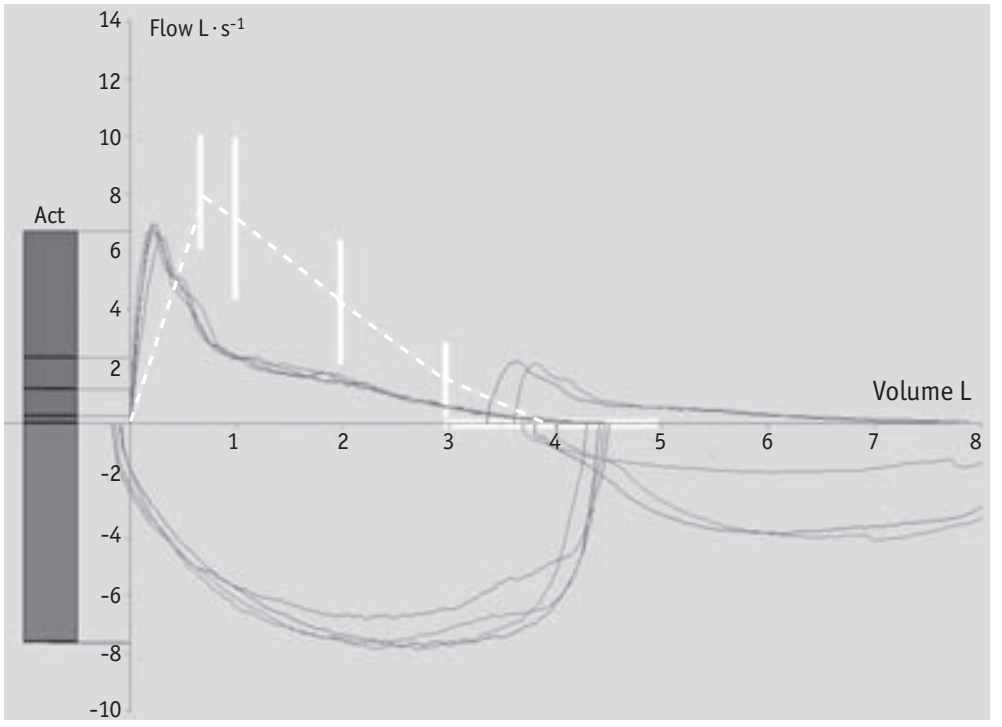

Figure 3

Moderate airflow limitation in a subject with asthma. Forced expiratory volume in $1 \mathrm{~s}$ (FEV 1 ): $73 \%$ predicted; forced vital capacity (FVC): $109 \%$ pred; FEV $1 / F V C$ ratio: $53 \%$. 
Table 2. Assessment of severity of airflow obstruction in chronic obstructive pulmonary disease according to forced expiratory volume $1 \mathrm{~s}\left(\mathrm{FEV} \mathrm{V}_{1}\right) \%$ predicted (\% pred)

\begin{tabular}{lc} 
FEV 1 \% pred & Category $^{\#}$ \\
\hline$\geqslant 80$ & Mild \\
\hline $50-79$ & Moderate \\
\hline $30-49$ & Severe \\
\hline$<30$ & Very severe \\
${ }^{\#}:$ values are post-bronchodilator and used in \\
combination with an $\mathrm{FEV}_{1} / \mathrm{FVC}$ ratio of $<0.7$.
\end{tabular}

adjusted for in predicted equations, since any reduction is abnormal.

Currently, a fixed ratio of $\mathrm{FEV}_{1} \%$ predicted is used to define normality, with $80 \%$ pred being the lower limit, but this may be incorrect in some populations. It has therefore been proposed that the 95th percentile for a population should be used for the limits of normality [4]. Reference equations and longitudinal data for this are awaited. Figure 2 shows an example of a normal flow-volume loop.

\section{Obstruction}

Obstruction is characterised by airflow limitation; there is a decreased airway calibre through

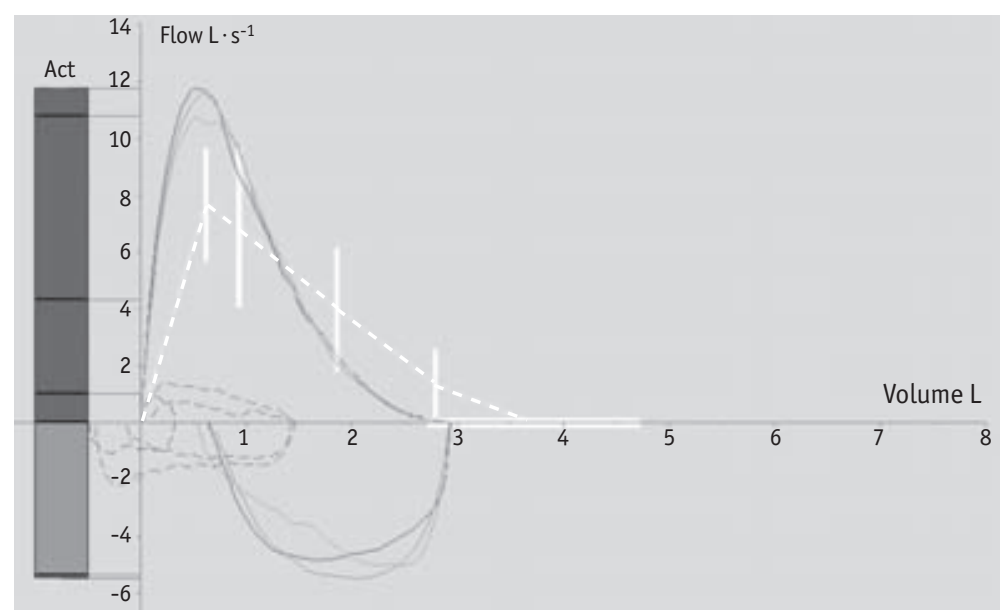

Figure 4

Example of a typical restrictive defect. Forced expiratory volume in $1 \mathrm{~s} \mathrm{(FEV} 1): 82 \%$ predicted; forced vital capacity (FVC): $85 \%$ pred; FEV 1 FVC: $84 \%$; peak expiratory flow: $157 \%$ pred. either smooth muscle contraction, inflammation, mucus plugging or airway collapse in emphysema.

Obstruction is characterised by:

- reduced $\mathrm{FEV}_{1}$;

- normal (or reduced) VC;

- normal or reduced FVC;

- reduced $\mathrm{FEV}_{\mathrm{1}} / \mathrm{FVC}$ ratio; and

- concave flow-volume loop (fig. 3).

Asthma is an obstructive disease but because it is reversible, spirometry may be normal when the person is not experiencing an exacerbation. Chronic obstructive pulmonary disease is also an obstructive disorder but tends not to be reversible in most cases. There are grades for the amount of obstruction seen [8], as shown in table 2 .

\section{Restriction}

Restrictive disorders are characterised by a loss in lung volume and are much rarer. This occurs in pulmonary fibrosis, pleural disease, chest wall disorders (kyphoscoliosis), neuromuscular disorders, pneumonectomy, pulmonary oedema and obesity, to name a few. Many so-called restrictive spirometry traces are due to failure to reach the end of expiration, falsely reducing FVC.

Restriction is characterised by:

- reduced FVC;

- normal-to-high $\mathrm{FEV}_{1} / \mathrm{FVC}$ ratio;

- normal looking shape on spirometry trace (fig. 4); and

- possibly a relatively high PEF.

\section{Mixed patterns}

If a person is a heavy smoker and has a fibrotic disease, for example, they may show a mixed picture on spirometry, which is harder to interpret. Further lung function tests may be useful in these cases to analyse static lung volumes (total lung capacity, functional residual capacity and reserve volume) and gas transfer (transfer factor of the lung for carbon monoxide).

\section{Spirometry in the workplace}

Certain occupations may involve exposure to respiratory irritants or sensitisers; in which 
case, surveillance is indicated, with pre-employment and regular (usually annual after the first year) spirometry. If accelerated lung function decline occurs, it is important to refer the worker to a specialist occupational clinic. If asthmatic symptoms occur (with or without the presence of lung function decline), simple spirometric measurements (usually PEF) should be performed 2-hourly from waking to going to bed, on days at work and away from work, for 4 weeks to assess any diagnostic patterns for occupational asthma [9]. PEFs should be completed using specialised forms that allow the worker to write down their work times, wake and sleep times, jobs done during the day and treatment taken. These can be downloaded from www.occupationalasthma.com. Once the worker has performed the measurements, they can be inputted in a computer program, such as Oasys (Occupational Asthma System; freely available for download from www.occupationa lasthma.com) [10-12] for analysis (fig. 5). When serial PEFs are of good quality, the mean pooled sensitivity for the diagnosis of occupational asthma is $82 \%$ and specificity is $89 \%$ [13].

$\mathrm{FEV}_{1}$ is the main measure used in specialised testing, such as specific inhalation challenge tests for the diagnosis of occupational asthma. Spirometry also aids in the diagnosis of lung diseases such as asbestosis, giving a measure of respiratory impairment for compensation or disability benefits.

\section{Training}

The key to getting useful, reproducible results is good training. Although written documents are useful, these should be used for reference rather than as a sole learning resource. It is essential to have practical training in spirometry to understand how to get the best from the patient and how to deal with the variety of patients you may come across. Several courses are available, including one run by the ARTP in association

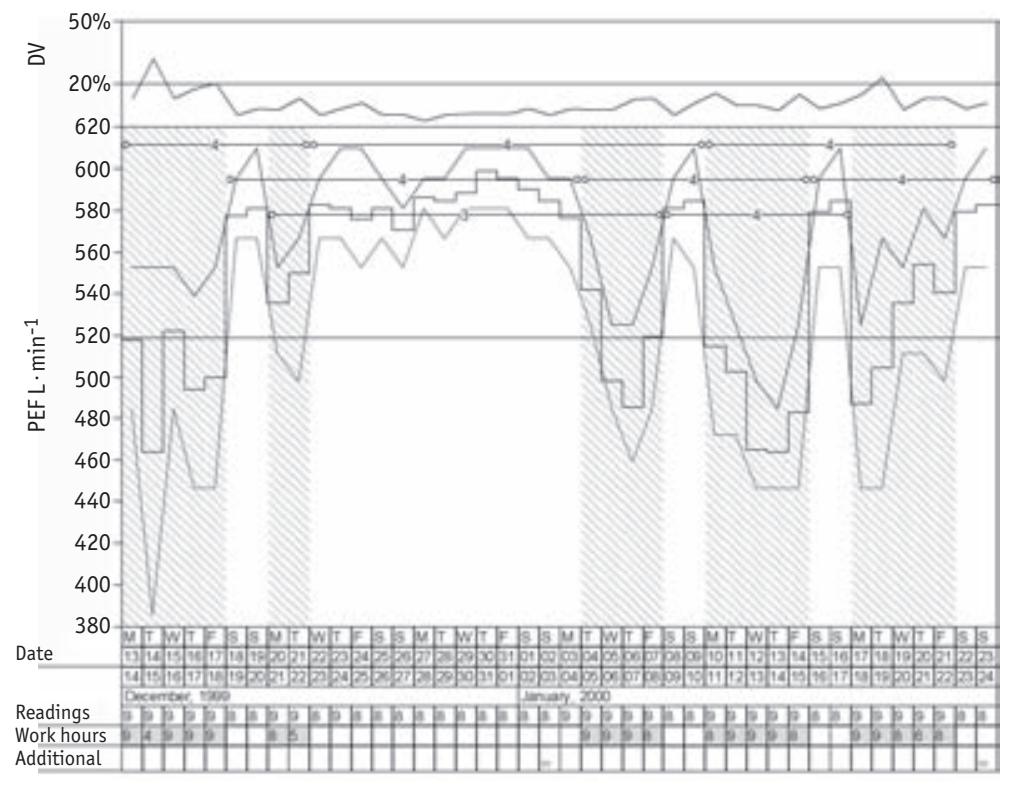

\section{Figure 5}

Serial peak flow plot showing occupational asthma in a worker exposed to formaldehyde. The top part of the plot shows the diurnal variation (DV), the middle section shows the highest peak expiratory flow (PEF), mean PEF and lowest PEF each day. Shaded areas are work days and white areas are days away from work. The Oasys complex scores (a complex is either a rest-work-rest or work-rest-work pattern) are shown and the predicted PEF line of $519 \mathrm{~L} \cdot \mathrm{min}^{-1}$. The bottom section shows the date, number of readings per day and number of hours worked. The overall Oasys score is 3.93 $(\geqslant 2.51$ is positive for occupational asthma). The area between curves score is

$80.4 \mathrm{~L} \cdot \mathrm{min}^{-1} \cdot \mathrm{h}^{-1}\left(\geqslant 15 \mathrm{~L} \cdot \mathrm{min}^{-1} \cdot \mathrm{h}^{-1}\right.$ is positive for occupational asthma) and time-point analysis is positive for occupational asthma.

with the BTS. Details can be found at www.artp. org.uk.

\section{Summary}

Spirometry is a useful measure for detecting early change in disease and provides physiological confirmation for diagnoses. When performed correctly, it can be used to assess disease progression and response to therapy. With the introduction of portable meters, it can be performed anywhere and by anyone with good training.
References

1. Miller MR, Hankinson J, Brusasco V, et al. Standardisation of spirometry. Eur Respir J 2005; 26: 319-338.

2. British Thoracic Society, Scottish Intercollegiate Guidelines Network. British Guideline on the Management of Asthma. Thorax. 2008; 63: Suppl. 4, iv1-iv121.

3. Global Initiative for Asthma. Global Strategy for Asthma Management and Prevention, New York: GINA, 2008.
4. Pellegrino R, Viegi G, Brusasco V, et al. Interpretative strategies for lung function tests. Eur Respir J 2005; 26: $948-968$.

5. Guidelines for the measurement of respiratory function. Recommendations of the British Thoracic Society and the Association of Respiratory Technicians and Physiologists. Respir Med 1994; 88: 165-194. 
6. Tweeddale PM, Alexander F, McHardy GJR. Short term variability in $\mathrm{FEV}_{1}$ and bronchodilator responsiveness in patients with obstructive ventilatory defects. Thorax 1987; 42: 487-490.

7. Quanjer PH. Standardization of lung function tests. Report of working party. Bull Eur Physiopathol Respir 1983; 19: 1-95.

8. National Clinical Guideline Centre. Chronic obstructive pulmonary disease: management of chronic obstructive pulmonary disease in adults in primary and secondary care, http://guidance.nice. org.uk/CG101/Guidance/pdf/English Date last accessed: 2010. Date last updated: January 18, 2012.

9. Nicholson PJ, Cullinan P, Taylor AJ, et al. Evidence based guidelines for the prevention, identification, and management of occupational asthma. Occup Environ Med 2005; 62: 290-299.
10. Gannon PFG, Newton DT, Belcher J, et al. Development of OASYS-2, a system for the analysis of serial measurements of peak expiratory flow in workers with suspected occupational asthma. Thorax 1996; 51: 484-489.

11. Moore VC, Jaakkola MS, Burge CBSG, et al. A new diagnostic score for occupational asthma; the area between the curves (ABC score) of PEF on days at and away from work. Chest 2009; 135: 307-314.

12. Burge CB, Moore VC, Pantin CF, et al. The diagnosis of occupational asthma from timepoint differences in serial PEF measurements. Thorax 2009; 64: $1032-1036$.

13. Moore VC, Jaakkola MS, Burge PS. A systematic review of serial peak expiratory flow measurements in the diagnosis of occupational asthma. Ann Respir Med 2010; 1: 31-44. 